\title{
Novel Method for the Direct Measurement of the $\tau$ Lepton Dipole Moments
}

\author{
J. Fu, ${ }^{1}$ M. A. Giorgi, ${ }^{2}$ L. Henry,${ }^{3}$ D. Marangotto, ${ }^{1}$ F. Martínez Vidal, ${ }^{3}$ A. Merli, ${ }^{1}$ N. Neri, ${ }^{1}$ and J. Ruiz Vidal ${ }^{3}$ \\ ${ }^{1}$ INFN Sezione di Milano and Università di Milano, 20133 Milano, Italy \\ ${ }^{2}$ INFN Sezione di Pisa and Università di Pisa, 56127 Pisa, Italy \\ ${ }^{3}$ IFIC, Universitat de València-CSIC, 46980 Valencia, Spain
}

(Received 7 February 2019; published 2 July 2019)

\begin{abstract}
A novel method for the direct measurement of the elusive magnetic and electric dipole moments of the $\tau$ lepton is presented. The experimental approach relies on the production of $\tau^{+}$leptons from $D_{s}^{+} \rightarrow \tau^{+} \nu_{\tau}$ decays, originating in fixed-target collisions at the LHC. A sample of polarized $\tau^{+}$leptons is kinematically selected and subsequently channeled in a bent crystal. The magnetic and electric dipole moments of the $\tau^{+}$ lepton are measured by determining the rotation of the spin-polarization vector induced by the intense electromagnetic field between crystal atomic planes. The experimental technique is discussed along with the expected sensitivities.
\end{abstract}

DOI: 10.1103/PhysRevLett.123.011801

Measurements of the electromagnetic dipole moments for common particles like the electron, muon, and nucleons, combined with precise theoretical calculations, provide stringent tests of physics within and beyond the standard model (SM) [1-8]. For short-lived particles like heavy baryons and the $\tau$ lepton, the short lifetime $\left(\sim 10^{-13} \mathrm{~s}\right)$ prevents the use of the spin-precession technique adopted in the muon $g-2$ experiment $[3,4]$. Recently, the possibility of directly measuring the electromagnetic dipole moments of short-lived baryons, produced in fixed-target collisions at the Large Hadron Collider (LHC) and channeled in bent crystals [9-14], has been considered. For the $\tau$ lepton, the use of $B^{+} \rightarrow \tau^{+} \nu_{\tau}$ decays was suggested [15] and more recently the $D_{s}^{+} \rightarrow \tau^{+} \nu_{\tau}$ process with higher yield has been explored [16]. In this Letter, a novel method that fully exploits the polarization properties of $\tau^{+}$leptons produced in $D_{s}^{+}$decays is proposed. The magnetic (MDM) and the electric (EDM) dipole moments are defined as $\boldsymbol{\mu}=g e \hbar /\left(2 m_{\tau} c\right) \mathbf{s} / 2$ and $\boldsymbol{\delta}=\operatorname{de} \hbar /\left(2 m_{\tau} c\right) \mathbf{s} / 2$, respectively, where $m_{\tau}$ is the $\tau$ mass, $g$ $(d)$ is the gyromagnetic (gyroelectric) factor, and $\mathbf{s}$ is the spinpolarization vector [17]. In the SM, the $\tau$ anomalous MDM is expected to be $a=(g-2) / 2 \approx 10^{-3}$ [18], and its EDM, $d$, to be minuscule [19]. However, the dipole moments can be largely enhanced in the presence of physics beyond the SM $[20,21]$. Methods based on precise measurements of the $\tau^{+} \tau^{-}$pair production cross section in $e^{+} e^{-}$annihilations set indirect limits on $a$ at the few percent level [22], still above

Published by the American Physical Society under the terms of the Creative Commons Attribution 4.0 International license. Further distribution of this work must maintain attribution to the author(s) and the published article's title, journal citation, and DOI. Funded by SCOAP. the SM prediction, and lead to limits on $\delta$ at the $10^{-16} e \mathrm{~cm}$ level [23]. Other indirect measurements have been suggested to improve the precision $[20,24,25]$.

The proposed solution to provide direct measurements of the $\tau$ dipole moments, illustrated in Fig. 1, is based on the large production cross section of high-energy polarized $\tau^{+}$ leptons, originating in proton fixed-target collisions at the LHC. The $\tau^{+} \rightarrow \pi^{+} \pi^{-} \pi^{+} \bar{\nu}_{\tau}\left(3 \pi \bar{\nu}_{\tau}\right)$ decay is considered. A bent crystal is employed to exploit the channeling phenomenon of positively charged particles aligned with the crystal atomic planes within a few $\mu \mathrm{rad}$. Angular momentum conservation selects negative helicity $\tau^{+}$leptons in the $D_{s}^{+}$rest frame. The $\tau^{+}$leptons emitted at relatively large $\theta_{y, D_{s} \tau}$ angles with respect to the $D_{s}^{+}$flight direction in the $y z$ plane show enhanced polarization along the $Y$ axis, perpendicular to the crystal plane. The Lorentz boost, making larger acceptance for forward- than for backward-emitted $\tau^{+}$leptons, induces a polarization of approximately $\beta^{\star} / \beta \approx 10 \%$ antialigned with the crystal $Z$ axis, where $\beta\left(\beta^{\star}\right)$ is the velocity of the $D_{s}^{+}\left(\tau^{+}\right)$in the laboratory

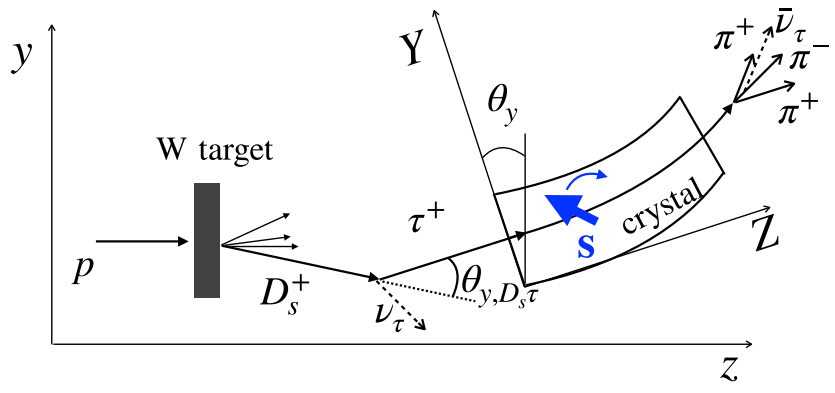

FIG. 1. Sketch of the fixed-target setup along with the $\tau^{+}$ production and decay processes (not to scale). The crystal frame $(X, Y, Z)$ is tilted in the laboratory frame $(x, y, z)$ by $\theta_{y}$ to avoid channeling of noninteracting protons. 
$\left(D_{s}^{+}\right.$rest) frame. Thus, the selection of the highest momentum candidates enhances the $Z$ polarization. The MDM (EDM) signature is given by the spin rotation in the $Y Z$ bending plane (appearance of a spin component along the $X$ axis) induced by the interaction with the crystal electric field. A novel analysis technique based on multivariate classifiers is employed to determine the rotation of the spin-polarization vector.

The vast majority of $\tau^{+}$leptons produced in proton fixedtarget collisions at $\sqrt{s} \approx 115 \mathrm{GeV}$ comes from $D_{s}^{+} \rightarrow \tau^{+} \nu_{\tau}$ decays. The corresponding production cross section $\sigma\left[p p \rightarrow D_{s}^{+}\left(\rightarrow \tau^{+} \nu_{\tau}\right) X\right] \approx 1.96 \mu \mathrm{b}$ is estimated using the rescaled charm production cross section measured by the LHCb experiment in proton-helium collisions at $\sqrt{s}=86.6 \mathrm{GeV}$ [26], the $c$ quark to $D_{s}^{+}$fragmentation fraction [27,28], and the $D_{s}^{+} \rightarrow \tau^{+} \nu_{\tau}$ branching fraction [29]. The conversion factor for a $7 \mathrm{TeV}$ proton on a $T=1 \mathrm{~cm}$ thick tungsten $(W)$ target to produce a $\tau^{+} \rightarrow 3 \pi \bar{\nu}_{\tau}$ final state is estimated

$$
\begin{aligned}
& \sigma\left[p p \rightarrow D_{s}^{+}\left(\rightarrow \tau^{+} \nu_{\tau}\right) X\right] N_{A} \frac{\rho T A_{N}}{A_{T}} \mathcal{B}\left(\tau^{+} \rightarrow 3 \pi \bar{\nu}_{\tau}\right) \\
& \quad \approx 2.1 \times 10^{-6},
\end{aligned}
$$

where $N_{A}$ is the Avogadro number, $\rho$ the target density, $A_{T}$ $\left(A_{N}\right)$ its atomic mass (mass number), and $\mathcal{B}\left(\tau^{+} \rightarrow 3 \pi \bar{\nu}_{\tau}\right)$ the $\tau^{+}$branching fraction [29].

In a reference frame defined by the crystal edges and comoving with the channeled particle, the $\tau^{+}$initial polarization $\mathbf{s}_{0}$ is given by the unit vector along the $D_{s}^{+}$ momentum in the $\tau^{+}$rest frame [30,31],

$$
\mathbf{s}_{0}=\frac{1}{\omega}\left(m_{\tau} \mathbf{q}-q_{0} \mathbf{p}+\frac{\mathbf{q} \cdot \mathbf{p}}{p_{0}+m_{\tau}} \mathbf{p}\right),
$$

where $\mathbf{p}(\mathbf{q})$ is the momentum of the $\tau^{+}\left(D_{s}^{+}\right)$and $p_{0}\left(q_{0}\right)$ its energy in the laboratory frame, $\omega=\left(m_{D_{s}}^{2}-m_{\tau}^{2}\right) / 2$, and $m_{D_{s}}$ is the $D_{s}^{+}$mass. The projections of $\mathbf{s}_{0}$ along the crystal frame axes are

$$
\begin{aligned}
& s_{0, X} \approx \frac{m_{\tau}|\mathbf{q}|}{\omega} \theta_{x, D_{s} \tau}, \\
& s_{0, Y} \approx \frac{m_{\tau}|\mathbf{q}|}{\omega} \theta_{y, D_{s} \tau}, \\
& s_{0, Z} \approx \frac{1}{\omega}\left(|\mathbf{q}| p_{0}-q_{0}|\mathbf{p}|\right),
\end{aligned}
$$

where $\theta_{x, D_{s} \tau}$ is the angle between the $D_{s}^{+}$and the $\tau^{+}$ momenta in the $x z$ plane. All angles are $\mathcal{O}\left(10^{-3}\right)$ rad due to the highly boosted $D_{s}^{+}$mesons and the small $D_{s}-\tau$ mass difference. Rotational invariance and the unconstrained $\theta_{x, D_{s} \tau}$ in the crystal $X Z$ plane imply a zero $s_{0, X}$ average.

Very large samples of fixed-target $D_{s}^{+} \rightarrow \tau^{+} \nu_{\tau}$ events are produced using Pythia [32], EvtGen [33], and a fast simulation that generates phase-space kinematics. The $\tau^{+}$ channeling is simulated using the parametrization and
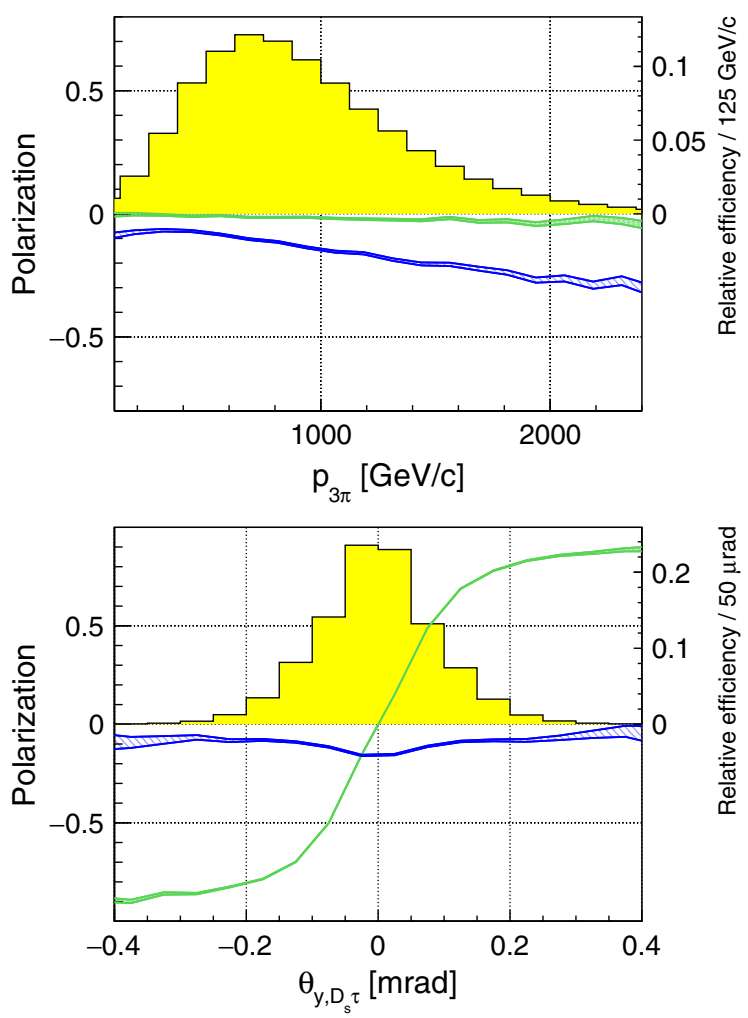

FIG. 2. Spin-polarization projections $s_{0, Z}$ (hatched blue bands) and $s_{0, Y}$ (solid green) as a function of $p_{3 \pi}$ (top) and $\theta_{y, D_{s} \tau}$ (bottom). The histograms, normalized to unity, show the spectra of channeled $\tau^{+}$. The bands represent 1 standard deviation regions.

procedures described in Refs. [13,34]. A polarized sample can be obtained by selecting channeled $\tau^{+}$and imposing kinematic requirements, as illustrated in Fig. 2 for the optimal experimental layout described later. For example, by requiring the $3 \pi$ system momentum, $p_{3 \pi}$, to exceed $1 \mathrm{TeV}$ an $s_{0, Z}$ polarization of about $-20 \%$ or higher is achieved. Instead, selecting regions of positive or negative $\theta_{y, D_{s} \tau}$ angles, in the following referred to as $\theta_{y}$ tagging, a large $s_{0, Y}$ polarization can be obtained.

The interaction of the MDM (EDM) of a relativistic charged particle channeled in a bent crystal induces spin precession $[10,13]$ in the bending plane (perpendicular to the bending plane). By measuring the spin-polarization components $s_{Y}$ and $s_{Z}$ ( $s_{X}$ component), it is possible to extract the MDM (EDM) information. In particular, the appearance of an $s_{X}$ component represents the EDM signature. The spinpolarization projections after precession in the crystal read

$$
\begin{aligned}
& s_{X} \approx-s_{0, Z} \frac{d^{\prime}}{a_{d}^{\prime}} \sin \Phi+s_{0, Y} \frac{d^{\prime} a^{\prime}}{a_{d}^{\prime 2}}(1-\cos \Phi), \\
& s_{Y} \approx s_{0, Z} \frac{a^{\prime}}{a_{d}^{\prime}} \sin \Phi+s_{0, Y}\left(\frac{d^{\prime 2}}{a_{d}^{\prime 2}}+\frac{a^{\prime 2}}{a_{d}^{\prime 2}} \cos \Phi\right), \\
& s_{Z} \approx s_{0, Z} \cos \Phi-s_{0, Y} \frac{a^{\prime}}{a_{d}^{\prime}} \sin \Phi,
\end{aligned}
$$


where $a^{\prime}=a+[1 /(1+\gamma)], d^{\prime}=d / 2, a_{d}^{\prime}=\sqrt{a^{\prime 2}+d^{\prime 2}}$, and $\Phi=\gamma \theta_{C} a_{d}^{\prime}$ is the precession angle, which is proportional to the $\tau^{+}$Lorentz factor $\gamma$ and the crystal bending angle $\theta_{C}$. Equation (4) holds at $\mathcal{O}\left(10^{-2}\right)$ precision, while expressions at $\mathcal{O}\left(10^{-5}\right)$ are reported in the Supplemental Material [35].

A technique based on multivariate classifiers is explored to extract the $\tau^{+}$polarization vector without prior knowledge of the detailed decay dynamics and of the $\tau^{+}$energy. A classifier discriminating between $\tau^{+}$with full positive $(+1)$ and negative $(-1)$ polarization along each crystal frame axis is built. The classifiers are trained on simulated events and are based upon variables describing the decay distribution. The used variables that provide sensitivity to the $\tau^{+}$spin polarization, referred to with the symbol $\zeta$, are the angles between the $3 \pi$ momentum in the $\tau^{+}$rest frame and the crystal frame axes, the angles describing the $3 \pi$ decay plane orientation in the $3 \pi$ rest frame with respect to the crystal frame axes, and two- and three-pion invariant masses. The $\tau^{+}$momentum is estimated by applying kinematic corrections, determined from simulated events, to the measured $p_{3 \pi}$ vector as a function of its magnitude and direction. In absence of the $\tau^{+}$production vertex, the flight direction is assumed to be that connecting the $D_{s}^{+}$ production vertex and the $\tau^{+}$decay vertex, lying in the crystal channeling plane. The vertex positions are smeared according to Gaussian distributions to mimic experimental resolutions, assumed to be $13 \mu \mathrm{m}(70 \mu \mathrm{m})$ for the production vertex in the longitudinal (transverse) direction with respect to the beam, and $100 \mu \mathrm{m}(1 \mathrm{~mm})$ for the decay vertex.

The polarization component $s_{i}$ along the $i$ th crystal frame axis $(i=X, Y, Z)$ is extracted by fitting the classifier distribution $\mathcal{W}_{i}(\eta)$ on data,

$$
\mathcal{W}_{i}(\eta)=\frac{1}{2}\left[\left(1+s_{i}\right) \mathcal{W}_{i}^{+}(\eta)+\left(1-s_{i}\right) \mathcal{W}_{i}^{-}(\eta)\right]
$$

where $\eta \equiv \eta(\zeta)$ is the classifier response, and $\mathcal{W}_{i}^{ \pm}(\eta)$ the templates representing the response for \pm 1 polarizations.

The statistical separation between templates also represents the squared average event information [39] of the polarization (at $s_{i}=0$ ) [40],

$$
S_{i}^{2}=\frac{1}{N_{\tau^{+}}^{\mathrm{rec}} \sigma_{i}^{2}}=\left\langle\left(\frac{\mathcal{W}_{i}^{+}(\eta)-\mathcal{W}_{i}^{-}(\eta)}{\mathcal{W}_{i}^{+}(\eta)+\mathcal{W}_{i}^{-}(\eta)}\right)^{2}\right\rangle,
$$

where $\sigma_{i}$ is the uncertainty on $s_{i}$, and $N_{\tau^{+}}^{\mathrm{rec}}$ is the number of channeled and reconstructed $\tau^{+}$. The template fit results for $s_{Y}$ polarization are shown in Fig. 3, while those for $s_{X}$ and $s_{Z}$ are shown in the Supplemental Material [35]. The estimated average event information is $S_{X} \approx S_{Y} \approx 0.42$ and $S_{Z} \approx 0.23$, using either Multilayer Perceptron Networks or Boosted Decision Trees [41], to be compared to the ideal value of 0.58 reached in case the complete kinematics of the $\tau^{+}$decay is reconstructed [40]. The difficulty in determining the $\tau^{+}$

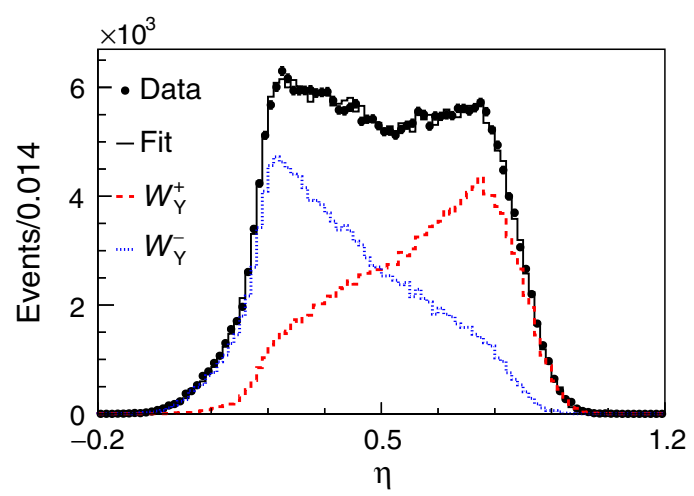

FIG. 3. Template fit results to the classifier response $\eta$ using simulated events for $s_{Y}$ polarization. The normalized separation between $\mathcal{W}_{Y}^{+}(\eta)$ (dashed red line) and $\mathcal{W}_{Y}^{-}(\eta)$ (dotted blue line) determines the average event information $S_{Y} \approx 0.42$.

momentum, due to the undetected $\bar{\nu}_{\tau}$, affects mainly the determination of the $s_{Z}$ polarization.

For small $\Phi\left(\right.$ as $\gamma \theta_{C} \sim 10$ and $\left.a_{d}^{\prime} \sim 10^{-3}\right)$ and $s_{0, Z}$ initial polarization, the statistical uncertainties on $a$ and $d$ are estimated from Eq. (4) as

$\sigma_{a} \approx \frac{1}{S_{Y} s_{0, Z} \gamma \theta_{C}} \frac{1}{\sqrt{N_{\tau^{+}}^{\mathrm{rec}}}}, \quad \sigma_{d} \approx \frac{2}{S_{X} s_{0, Z} \gamma \theta_{C}} \frac{1}{\sqrt{N_{\tau^{+}}^{\mathrm{rec}}}}$

For $s_{0, Y}$ initial polarization,

$\sigma_{a} \approx \frac{1}{S_{Z} s_{0, Y} \gamma \theta_{C}} \frac{1}{\sqrt{N_{\tau^{+}}^{\mathrm{rec}}}}, \quad \sigma_{d} \approx \frac{2}{S_{X} s_{0, Y}\left(\gamma \theta_{C}\right)^{2} a^{\prime}} \frac{1}{\sqrt{N_{\tau^{+}}^{\mathrm{rec}}}}$,

which show comparable sensitivity to $a$ but disfavored by a factor $1 /\left(\gamma \theta_{C} a^{\prime}\right) \sim 100$ to $d$ with respect to Eq. (7) for initial $s_{0, Z}$ polarization.

The optimization of the experimental layout is performed using simulated events for the case of initial $s_{0, Z}$ polarization. The region of minimal uncertainty on $a$ and $d$ is determined using a scan in the $\left(\theta_{C}, L, \theta_{y}, L_{\mathrm{tar}}\right)$ parameter space, where $\theta_{C}(L)$ is the crystal bending angle (length) and $L_{\mathrm{tar}}$ the distance between the target center and crystal upstream edge. Channeled $\tau^{+}$are required to have $p_{3 \pi}>$ $800 \mathrm{GeV} / c$ to enhance $s_{0, Z}$ polarization, and to originate before the crystal and to decay after the crystal to insure maximum $\Phi$ precession angle. For a $\mathrm{Ge}(\mathrm{Si})$ crystal tilted by $\theta_{y}=0.1 \mathrm{mrad}$, the optimal parameters $\theta_{C} \approx 16 \mathrm{mrad}$, $L \approx 8(11) \mathrm{cm}$, and $L_{\mathrm{tar}} \approx 12 \mathrm{~cm}$ are obtained (see Supplemental Material [35]). The Ge crystal provides relatively high channeling efficiency, $\approx 6.3 \times 10^{-6}$, a factor of 3 higher than for Si. Recently, crystal prototypes with similar length and bending angle have been tested on beam at the CERN SPS [42]. The selected $\tau^{+}$sample has $s_{0, Z} \approx-18 \%, s_{0, Y} \approx 0 \%$ polarization, and average Lorentz factor $\gamma \approx 800$. A $s_{0, Y} \approx \mp 40 \%$ polarization can be achieved with a $\theta_{y}$ tagging that discriminates between 


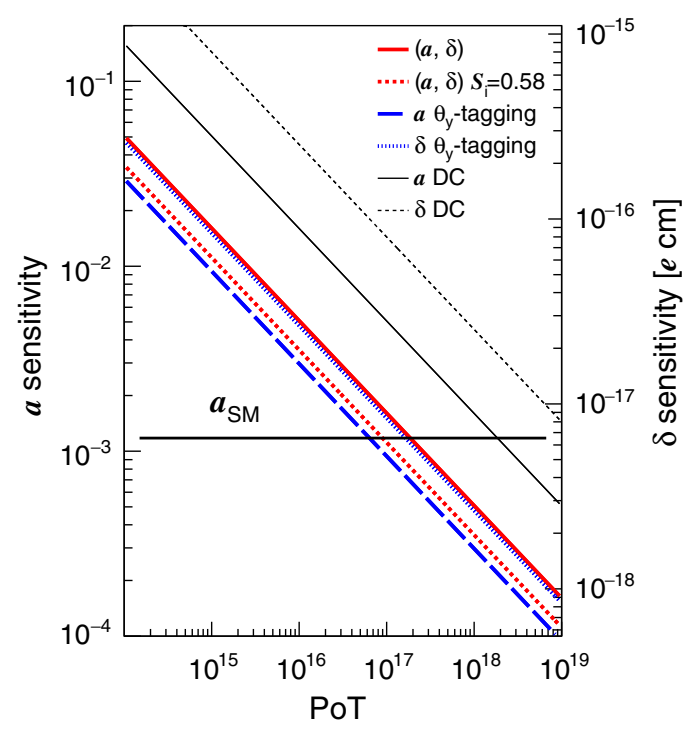

FIG. 4. Estimated sensitivities for $a$ and $\delta$ as a function of the number of protons on a $2.5 \mathrm{~cm}$ thick $\mathrm{W}$ target (PoT) for a $\mathrm{Ge}$ crystal with optimal parameters (thick solid red line), compared to other configurations (see text). These are labeled as $(a, \delta)$ when the corresponding lines overlap. The SM model prediction for $a$ [18] is also indicated.

positive and negative $\theta_{y, D_{s} \tau}$ angles. Information statistically correlated with $\theta_{y, D_{s} \tau}$ is required for $\theta_{y}$ tagging. A possible strategy could be the exploitation of the global event topology, e.g., kinematic distributions of particles associated with the interaction point where the $D_{s}^{+}$is produced. The relatively large separation between the target and the crystal would allow for additional instrumentation; e.g., several layers of pixel radiation-hard diamond sensors could be used to reconstruct the $D_{s}^{+}$trajectory. Another possibility would be to place a second bent crystal to channel the $D_{s}^{+}$using a layout similar to that suggested in Ref. [16], inducing $s_{0, Y} \approx \mp 60 \%$ for tagged events with an efficiency of a few percent.

Dipole moment sensitivities are assessed from a large number of pseudoexperiments generated and fit using a probability density function based on the spin precession equation of motion reported in Eq. (4), and the classifier distributions in Eq. (5). Figure 4 illustrates the estimated sensitivities as a function of the number of impinging protons for a Ge crystal with optimal parameters (thick solid red line). Sensitivities for other configurations with maximum average event information $S_{i}=0.58$ (thick dotted red line), $\theta_{y}$ tagging based on a discrimination between positive and negative $\theta_{y, D_{s} \tau}$ with ideal tagging efficiency of 100\% (thick dashed and hatched blue lines), and the double crystal (DC) option proposed in Ref. [16] (thin solid and dotted black lines), are also shown for comparison. A detector reconstruction efficiency of $40 \%$ is assumed. The corresponding sensitivities for $\mathrm{Si}$ are about a factor of 2 worse.
The channeling process keeps the high momentum unchanged while deflecting the $\tau^{+}$at the bending angle $\theta_{C} \approx 16 \mathrm{mrad}$. This signature can be identified in the $3 \pi \bar{\nu}_{\tau}$ decays through the reconstruction of the $3 \pi$ vertex and momentum. For highly boosted particles with $\gamma \approx 800$ the latter defines the $\tau^{+}$direction with an uncertainty of $\approx 0.5 \mathrm{mrad}$, mainly due to the missing $\bar{\nu}_{\tau}$. The contribution of nonchanneled leptons is reduced to a negligible level $<0.3 \%$ using the following selection criteria: $p_{3 \pi} \geq 800 \mathrm{GeV} / c, 3 \pi$ momentum direction consistent with $\theta_{C}$ within $1.5 \mathrm{mrad}$, and the $3 \pi$ vertex located after the crystal, at a distance $L+L_{\mathrm{tar}} \gtrsim 20 \mathrm{~cm}$ from the interaction point. With these requirements, $28 \%$ of the $\tau^{+}$candidates are channeled through a fraction of the crystal length. These are mainly events in which the $D_{s}^{+}$decays inside the crystal or the $\tau^{+}$does not reach the end of the crystal, either because it decays or is dechanneled. Nevertheless, only $\tau^{+}$ particles that travel almost through the entire crystal are selected. They experience a very similar electromagnetic field, inducing a relatively small bias on the spin precession angle $\Phi$ of $1.4 \%$ that can be corrected. Background contributions from channeled hadron decays with $3 \pi$ in the final state, e.g., $D^{+}, D_{s}^{+}$mesons, $\Lambda_{c}^{+}$baryons, can be vetoed using the reconstructed invariant mass and event information from a dedicated detecting apparatus. Systematic effects could arise from the limited knowledge of the crystal position and orientation, the initial polarization, and the $\tau^{+}$momentum. Those uncertainties can be controlled using up- and down-bending crystals, inducing opposite spin precession [13], by reconstructing unchanneled $\tau^{+}$decays with kinematic properties similar to the signal, and by using detailed simulations of the experimental setup calibrated with data. Possible effects due to $\tau^{+}$ weak interactions with the crystal are estimated to be negligible [14] compared to the sensitivity and can be removed by using different crystal bending orientations.

In summary, a novel method for the direct measurement of the $\tau$ MDM and EDM has been presented with interesting perspective for a stringent test of the SM and search of new physics. The fixed-target setup and the analysis technique have been discussed along with sensitivity projections for possible future scenarios. The SM prediction for the $\tau$ MDM could be verified experimentally with a sample of around $10^{17} \mathrm{PoT}$, whereas at the same time a search for the $\tau$ EDM at the level of $10^{-17} e \mathrm{~cm}$ or below could be performed. This would require about $10 \%$ of the protons stored during a decade of LHC operation [43]. In preparation of a possible future experiment this method could be tested using the fixed-target setup proposed for the study of heavy baryons $[10,12,13]$ with the $\mathrm{LHCb}$ apparatus. The possibility of a test or an experiment at the CERN SPS will also be explored.

We express our gratitude for stimulating discussions to V. Baryshevsky, F. J. Botella, G. Cavoto, A. S. Fomin, A. Mazzolari, A. Pich, and J. Walsh. We acknowledge 
support from INFN (Italy), Ministerio de Economía y Competitividad, and Generalitat Valenciana (Spain), the Severo Ochoa excellence certification SEV-2014-0398-01, and the ERC Consolidator Grant SELDOM G.A. 771642.

[1] P. J. Mohr, D. B. Newell, and B. N. Taylor, Rev. Mod. Phys. 88, 035009 (2016).

[2] V. Andreev et al. (ACME Collaboration), Nature (London) 562, 355 (2018).

[3] G. W. Bennett et al. (Muon (g-2) Collaboration), Phys. Rev. D 73, 072003 (2006).

[4] G. W. Bennett et al. (Muon (g-2) Collaboration), Phys. Rev. D 80, 052008 (2009).

[5] J. M. Pendlebury et al., Phys. Rev. D 92, 092003 (2015).

[6] G. Schneider et al., Science 358, 1081 (2017).

[7] B. Sahoo, Phys. Rev. D 95, 013002 (2017).

[8] B. Graner, Y. Chen, E. G. Lindahl, and B. R. Heckel, Phys. Rev. Lett. 116, 161601 (2016); 119, 119901(E) (2017).

[9] V. G. Baryshevsky, Phys. Lett. B 757, 426 (2016).

[10] F. J. Botella, L. M. Garcia Martin, D. Marangotto, F. Martinez Vidal, A. Merli, N. Neri, A. Oyanguren, and J. Ruiz Vidal, Eur. Phys. J. C 77, 181 (2017).

[11] V. G. Baryshevsky, Nucl. Instrum. Methods Phys. Res., Sect. B 402, 5 (2017).

[12] A. S. Fomin, A. Y. Korchin, A. Stocchi, O. A. Bezshyyko, L. Burmistrov, S. P. Fomin, I. V. Kirillin, L. Massacrier, A. Natochii, P. Robbe et al., J. High Energy Phys. 08 (2017) 120.

[13] E. Bagli, L. Bandiera, G. Cavoto, V. Guidi, L. Henry, D. Marangotto, F. Martinez Vidal, A. Mazzolari, A. Merli, N. Neri et al., Eur. Phys. J. C 77, 828 (2017).

[14] V. G. Baryshevsky, Eur. Phys. J. C 79, 350 (2019)..

[15] M. A. Samuel, G.-w. Li, and R. Mendel, Phys. Rev. Lett. 67, 668 (1991); 69, 995(E) (1992).

[16] A. S. Fomin, A. Y. Korchin, A. Stocchi, S. Barsuk, and P. Robbe, J. High Energy Phys. 03 (2019) 156.

[17] E. Leader, Spin in Particle Physics, Cambridge Monographs on Particle Physics, Nuclear Physics and Cosmology Vol. 15 (Cambridge University Press, Cambridge, England, 2011).

[18] S. Eidelman and M. Passera, Mod. Phys. Lett. A 22, 159 (2007).

[19] M. Pospelov and A. Ritz, Phys. Rev. D 89, 056006 (2014).

[20] A. Pich, Prog. Part. Nucl. Phys. 75, 41 (2014), and references therein.

[21] W. Dekens, J. de Vries, M. Jung, and K. K. Vos, J. High Energy Phys. 01 (2019) 069.

[22] J. Abdallah et al. (DELPHI Collaboration), Eur. Phys. J. C 35, 159 (2004).
[23] K. Inami et al. (Belle Collaboration), Phys. Lett. B 551, 16 (2003).

[24] A. Hayreter and G. Valencia, J. High Energy Phys. 07 (2015) 174.

[25] X. Chen and Y. Wu, arXiv:1803.00501.

[26] R. Aaij et al. (LHCb Collaboration), Phys. Rev. Lett. 122, 132002 (2019).

[27] M. Lisovyi, A. Verbytskyi, and O. Zenaiev, Eur. Phys. J. C 76, 397 (2016).

[28] L. Gladilin, Eur. Phys. J. C 75, 19 (2015).

[29] C. Patrignani et al. (Particle Data Group), Chin. Phys. C 40, 100001 (2016).

[30] F. Halzen and A. D. Martin, Quarks and Leptons: An Introductory Course in Modern Particle Physics (Wiley, New York, 1984).

[31] V. B. Berestetskii, E. M. Lifshitz, and L. P. Pitaevskii, Quantum Electrodynamics, Course of Theoretical Physics Vol. 4 (Butterworth-Heinemann, Oxford, 1982).

[32] T. Sjostrand, S. Mrenna, and P. Z. Skands, J. High Energy Phys. 05 (2006) 026.

[33] D. J. Lange, Nucl. Instrum. Methods Phys. Res., Sect. A 462, 152 (2001).

[34] V. M. Biryukov et al., Crystal Channeling and Its Application at High-Energy Accelerators (Springer-Verlag, Berlin, 1997).

[35] See Supplemental Material at http://link.aps.org/ supplemental/10.1103/PhysRevLett.123.011801 for spinpolarization precession expressions at $\mathcal{O}\left(10^{-5}\right)$ precision, template fit results for $s_{X}$ and $s_{Z}$ polarizations, and regions of minimal uncertainty on $a$ and $d$ as a function of the experimental layout parameters, which includes Refs. [36-38].

[36] L. H. Thomas, Nature (London) 117, 514 (1926).

[37] L. H. Thomas, Philos. Mag. 3, 1 (1927).

[38] V. Bargmann, L. Michel, and V. L. Telegdi, Phys. Rev. Lett. 2, 435 (1959).

[39] M. Kendall, A. Stuart, and J. Ord, The Advanced Theory of Statistics (Charles Griffin, London, 1983).

[40] M. Davier, L. Duflot, F. Le Diberder, and A. Rouge, Phys. Lett. B 306, 411 (1993).

[41] H. Voss, A. Hoecker, J. Stelzer, and F. Tegenfeldt, Proc. Sci. ACAT2007 (2007) 040, https://pos.sissa.it/050/040/pdf; A. Hoecker, P. Speckmayer, J. Stelzer, J. Therhaag, E. von Toerne, H. Voss, M. Backes, T. Carli, O. Cohen, et al., arXiv:physics/0703039.

[42] A. Mazzolari (private communication).

[43] G. Apollinari, I. Bejar Alonso, O. Bruning, P. Fessia, M. Lamont, L. Rossi, and L. Tavian, High-Luminosity Large Hadron Collider (HL-LHC) Technical Design Report V. 0.1 (CERN, Geneva, 2017), Vol. 4, pp. 1-24, https://doi.org/10 .23731/CYRM-2017-004. 